www.jmscr.igmpublication.org Impact Factor 5.244

Index Copernicus Value: 5.88

ISSN (e)-2347-176x ISSN (p) 2455-0450

crossref DOI: http://dx.doi.org/10.18535/jmscr/v4i6.56

Journal Of Medical Science And Clinical Research

An Official Publication of IGM Publication

\title{
Role of Single Dose Antibiotic Prophylaxis in Clean General Surgery
}

\author{
Dr Mahendra Bendre ${ }^{1}$, Dr Vinayak Kshirsagar ${ }^{2}$, Dr Prashant Male ${ }^{3}$, \\ Dr Sarang Rathod ${ }^{4}$, Dr Shivmurti Khandalkar ${ }^{5}$ \\ ${ }^{1}$ Professor, D.Y. Patil Medical College, Pimpri, Pune-411018 \\ Email: mahendrabendredr@gmail.com, Mobile no-9561744365 \\ ${ }^{2}$ Assistant Professor, D.Y. Patil Medical College, Pimpri, Pune-411018 \\ Email:drvinusagar@yahoo.co.in, Mobileno. - 9850930621 \\ ${ }^{3}$ Assistant Professor, D.Y.Patil Medical College, Pimpri, Pune-411018 \\ Email: drprashant.male@gmail.com, Mobile no. - 7057145363 \\ ${ }^{4}$ Senior Resident, D.Y.Patil Medical College, Pimpri, Pune-411018 \\ Email: cr7.zizou.kaka@gmail.com, Mobile no._- 7769927522 \\ ${ }^{5}$ Assistant Professor, D.Y.Patil Medical College, Pimpri, Pune-411018 \\ Email: drshiva.khandalkar@gmail.com, Mobile no. - 9422636996
}

\begin{abstract}
Background - Postoperative wound infection is an important cause of morbidity in surgical patients and antibiotic prophylaxis forms one of the important preventive measures.

Aims and Objectives - This study was performed in a major general hospital to establish the efficacy of antibiotic prophylaxis in clean general surgeries.

Materials and Methods - The study was done in 100 cases divided in 2 groups, one of which was given a single dose of ceftriaxone within 2 hours of operative incision without any postoperative antibiotics and the other was given routine postoperative antibiotics. Postoperative evaluation was done for development of fever or wound infection.

Observation and Results - Mean age was 35 and 33 in both groups while mean operative time was 45 - 53 minutes. Majority of cases performed were of hernioplasty. Various other factors such as length of incision, use of electrocautery and postoperative hospital stay were considered. Incidence of infection in both groups was similar but cost reduction of $88.11 \%$ was achieved in the first group.

Conclusion - The restriction of antibiotic usage in preoperative period and omission of it in postoperative period didn't result in increase in incidence of surgical site infection. However significant cost reduction is achieved in the cases where preoperative prophylactic antibiotics were used and hence can be advocated in clean general surgeries.

Key Words: Preoperative, Antibiotic, Prophylaxis, Clean, Postoperative, Infection
\end{abstract}

\section{INTRODUCTION}

Postoperative wound infections have an enormous impact on patients' quality of life and contribute substantially to the cost of patient care. It is a major source of morbidity and mortality in surgical patients.

The potential consequences range from increased pain and care of an open wound to sepsis and even 
death. Approximately 1 million patients have such wound infections each year in the United States, extending hospital stay and increasing cost of hospitalization by $20 \%$. The impact of such a sepsis doubles in our scenario as the patient has to lose his livelihood for the period of hospitalization.

The process depends on the interaction of various host, local tissue and microbial virulence factors. Measures intended to prevent infection include preoperative optimization of illness, control of the operative environment, proper cleansing of the skin and use of aseptic surgical technique. Preventing microbial penetration, reducing the microbial inoculums and treating established infection have been important developments. But the efficacy and impact of antimicrobial prophylaxis has clearly been demonstrated to be significant ${ }^{(1,2)}$. Multiple clinical trials have showed systemic antibiotics to be highly effective when used just before, during and immediately after an operation.

This study endeavours to find whether antibiotic prophylaxis improves outcome in a hospital set up of a developing country, whether it is feasible in such a set up and whether it is cost effective.

\section{AIMS AND OBJECTIVES}

1. To study proportion of early postoperative infection after single dose antibiotic prophylaxis in clean general surgeries.

2. To study proportion of early post operative infection after multiple dose post operative antibiotics in clean general surgeries.

3. To compare proportion of early post operative infection in clean surgeries after single dose of prophylactic antibiotic and multiple dose post operative antibiotics.

\section{MATERIALS AND METHODS}

This interventional, quasi-experimental study was conducted in a teaching general hospital over a period of 3 years. Sample size was 100 patients, equally divided into two groups A and B. Patients of either age \& gender undergoing clean general surgery were followed up till one month. Patients with generalized debilitating disease, diabetes mellitus, infective focus, allergy to cephalosporin and h/o use of antibiotics within last 7 days were excluded from the study.

The protocol was approved by hospital ethical committee and written informed consent was obtained from all patients. Ceftriaxone, (a third generation cephalosporin) was selected for antibiotic prophylaxis because of its broad spectrum, long half life and low toxicity. Patients in group A were given only single dose of 1 gram Ceftriaxone (50 $\mathrm{mg} / \mathrm{kg}$ body weight intravenously within 2 hours of the operative incision and did not receive post operative antibiotics. Patients who underwent appendicectomy received single dose of Metronidazole preoperatively $(1.5 \mathrm{cc} / \mathrm{kg}$ body weight) in addition. Patients in B group were given routine postoperative antibiotics.

Evaluation was done using the prescribed proforma for post operative fever developing or persisting 48 hours after the surgery, discharge from the wound and overlying skin inflammation. Wound was examined on $3^{\text {rd }}, 8^{\text {th }}, 15^{\text {th }}$, and $28^{\text {th }}$ postoperative day. Bacteriological examination was done to confirm the diagnosis if there was any evidence of infection and was managed according to culture and sensitivity report. Results were tested by Chi-square test. A p- value $<0.05$ was considered as statically significant.

\section{RESULTS}

In this study 50 patients were included in the each case \& control group.

(1) Age: The youngest patient was of $2 \mathrm{yrs}$ and oldest was of $75 \mathrm{yrs}$. The maximum numbers of cases were in the age group of 21-30 (14 and 13 respectively). There were only 2 cases in age group of $71-80$. The mean age was 35 and 33 years in both groups and $\mathrm{P}$ value was 0.6235 .

(2) Sex: There were 39 males and 11 females in case group. 45 males and 5 females were present in the control group. 
(3) Body Mass Index: The mean BMI was 21.18 and 20.41 in the case and control group respectively. Applying $\mathrm{z}$ test, the difference between the groups was considered statistically insignificant.

(3) Mean Hemoglobin levels: Mean Hemoglobin level was 11.56 and 11.89 in both groups and $\mathrm{p}$ value $(0.25)$ was comparable.

(4)Mean operative time (minutes):

\begin{tabular}{|l|l|l|l|}
\hline $\begin{array}{l}\text { Mean } \\
\text { operative } \\
\text { time }\end{array}$ & Case & Control & P value \\
\cline { 2 - 4 } & 53.9 & 45.3 & 0.080162 \\
\hline
\end{tabular}

Mean operative time was comparable in both groups.

(5) Surgical procedures:

\begin{tabular}{|l|l|l|}
\hline Procedure & Case & Control \\
\hline Appendicectomy & 2 & 5 \\
\hline Circumcision & 4 & 3 \\
\hline Herniotomy & 4 & 6 \\
\hline Excision \& biopsy & 10 & 3 \\
\hline Enucleation & 4 & 1 \\
\hline Jaboulay's repair & 3 & 1 \\
\hline Herniorrhaphy & 2 & \\
\hline Hernioplasty & 21 & 30 \\
\hline
\end{tabular}

Majority of cases in both groups were Hernioplasty.

(6) Time of administration of prophylactic antibiotic: All cases were given preoperative dose of antibiotic within two hours of incision. $40 \%$ of patients received dose within 30- $60 \mathrm{~min}$.

(7)Intraoperative electrocautery: Electrocautery was used in 28 cases in case group \& 34 cases in control group. The $\mathrm{p}$ value was 0.3030 which was not significant.

(8) Post operative stay: The mean hospital stay was 6.04 and 7.24 in case and control groups respectively and the difference was insignificant.

(9) Mean cost of antibiotics used: Mean cost of antibiotic was Rs 65.80 in case group and Rs 553.60 in control group, showing a cost reduction of $88.11 \%$.

(10) Surgical site infection: The incidence of infection in both groups was similar and $p$ value was statistically insignificant.

\begin{tabular}{|l|l|l|l|}
\hline Presence of SSI & Case & Control & P value \\
\hline Yes & 3 & 4 & 1.00 \\
\cline { 1 - 3 } No & 47 & 46 & \\
\hline
\end{tabular}

\section{DISCUSSION}

Use of antibiotic in all types of surgeries has considerably decreased morbidity and mortality. Prophylactic antibiotics decrease postoperative morbidity, shorten hospitalization and reduce the overall cost attributable to infections. In a study performed at the time when antibiotic prophylaxis was usually initiated postoperatively, the infection rate for clean wounds was $5.1 \%$ and based on a prospective study of 20,000 wounds where preoperative antibiotics was used, it was $0.8 \%{ }^{(3)}$. Overuse of antibiotics results in the emergence of resistant organisms, causes great economic burden on the health system ${ }^{(4,5)}$, increases risk of side effects, allergic reactions, drug interactions and thrombophlebitis and is often discouraged ${ }^{(5,6)}$.

It is proved that incorrect timing of surgical prophylaxis is associated with 2-6 fold increase in rate of $\mathrm{SSI}^{(7)}$. Still it is being practiced by surgeons in developing world for fear of infection and postoperative morbidity. This seems reasonable in clean- contaminated, contaminated and dirty cases. However the infection rates are low in these cases even in developing countries. $(8,9)$.

Some studies mention that there is no need of antibiotic prophylaxis in clean surgical cases. But majority of these studies are carried out in developed countries where the scenario is different. Some studies mention that antibiotic prophylaxis is recommended for operations with high risk of postoperative wound infection ${ }^{(10)}$. The efficacy of antibiotic prophylaxis in clean surgeries depends greatly on obtaining adequate levels of antibiotic in the tissue at risk ${ }^{(11)}$. There is no rationale for post operative antibiotic administration in clean cases with no evidence of wound infection.

A tailor made and rationale approach determination was the primary goal of this study. We used a case control model with random sampling. The prophylactic antibiotic was chosen to be Ceftriaxone considering its broad spectrum, long $\mathrm{t} 1 / 2$ and good efficacy. A meta-analysis published by Silvano Esposito (2004) 
recommends Ceftriaxone as superior prophylaxis antibiotic ${ }^{(12)}$. Woodfield JC, et al. (2002) had also found Ceftriaxone to be versatile choice, although expensive. It is particularly effective against Staphylococcus Aureus, has long half life and no active metabolites ${ }^{(13)}$.

We prospectively compared two sets of patients on the basis of vital parameters viz age, gender, height and weight, in case and control groups and evaluated them for presence of surgical site infections. Other factors directly or indirectly affecting the occurrence of surgical site infections were also considered.

Rey et al reported that the development of surgical site infection was significantly associated with the duration of surgery ${ }^{(14)}$. Increase in operative time not only increases the risk of infection but also increases the need for repeating the dose of prophylactic antibiotic. For longer procedures, readministration of the drug is indicated at intervals of one or two times the half life of the drug ${ }^{(1,15)}$. In our study the difference in the operative time in both groups was statistically insignificant and mean operative time in both groups did not warrant use of repeat dose. The maximum operative time was 120 minutes. Thus, single dose of preoperative Ceftriaxone is sufficient to cover the incision till the end of surgery.

The primary cause of infection of surgical wound is the endogenous bacteria harboured by the patients on their skin ${ }^{(16)}$ and the practice of preoperative bath, preoperative shaving have a profound effect on the infection rates. Preoperative bath was taken by majority of patients in both groups and the difference were statistically insignificant. Preoperative cleansing with povidone iodine and ethanol was done in all patients.

It is recommended that the prophylactic antibiotic should be administered within two hours before the incision ${ }^{(17,1,13)}$, as is done in our study where the mean time is 55 minutes before incision. Failure to administer the first dose of antibiotic within the two hour window before incision (to achieve adequate blood levels of the antibiotic during surgery) remains a common error, occurring in 27 to $54 \%$ of all operations in a New York study ${ }^{(18)}$. Although higher SSI rates were observed by Mary T. Hawn for timing of antibiotic given more than 60 minutes before incision, risk is not significantly affected by the timing ${ }^{(19)}$.Most optimal time of administration is found to be $1 / 2$ hour before incision when the infection rate has been found to be $<2 \%$, as compared to the administration 1 hour before when it is $3 \%{ }^{(20)}$.

Length of incisions used were comparable in both groups under observation. Intraoperative use of electrocautery creates local ischemic environment and increases seroma formation and was also comparable in both the groups. Use of mesh during surgery makes the use of antibiotic essential considering the catastrophic implications of mesh infection. According to Rey et al. the use of wound drains also increases the risk of infection ${ }^{(14)}$. Both the groups were found to be comparable in this regard in our study.

Post operative stay in both groups was statistically insignificant and study could not prove a reduction in days of hospitalization. Patients could not be discharged till suture removal as proper wound care and stitch removal cannot be ensured at periphery. Therefore preoperative uses of antibiotic does not reflect in reduction of hospital stay in our scenario.

Mean cost of antibiotic used in control group was Rs. 553.60 and that in case group was Rs. 65.80. This signifies almost $88.11 \%$ cost reduction by single dose preoperative prophylaxis which goes a long way in reducing health care cost. The surgical site infection in study group was $6 \%$ and in control group was $8 \%$. J. Wesley Alexander has shown that use of prophylactic antibiotic decreased the infection rate by half and has clear benefit in clean elective operations such as hernias and breast surgeries ${ }^{(21)}$.

The study could not establish a significant difference in outcome between the 2 groups as the incidence of surgical site infection were 
equivocal. This is in line with the observations of Cochrane meta-analysis, which states that antibiotic prophylaxis (especially in clean surgeries) cannot be firmly recommended or discarded. However the restriction of antibiotic usage to preoperative period and omission of antibiotic in post operative period did not amount to increase in the incidence of surgical site infections and is also cheaper. A short term antibiotic prophylaxis of one preoperative and 2 postoperative doses of antibiotics is advocated by some (22), which prevents the emergence of resistant strains and is cost effective also.

\section{CONCLUSION}

The study endeavours to find out whether preoperative antibiotic prophylaxis in clean general surgeries improves outcomes in a hospital set up as regards to postoperative wound infections. There was no statistically significant difference in the proportion of early postoperative wound infections between the patients who received single dose of ceftriaxone and patients who received routine postoperative antibiotics in clean general surgeries. Thus it is a feasible and cost effective measure even in a set up of developing countries and can be well advocated.

\section{REFERENCES}

1. Dellinger EP, Gross PA et al - 'Quality standard for antimicrobial prophylaxis in surgical procedures' - Cli Infec Diseases 1994; 18: 422-7

2. Platt R, Zuker JR ET AL - 'Prophylaxis against wound infection following herniorraphy or breast surgery' - Jour. Infec. Diseases 1992; 166: 556-60

3. Advoc commite on trauma, National research council division of medical sciences - 'Postoperative wound infections : Influence of ultraviolate irradiation of operating room and varios other factors' Ann. Surg 1964; $160: 2$

4. Dar LR, Fayaz F - 'Prophylactic antibiotics in elective major gynaecological surgery : single perioperative dose vs multiple postoperative doses' Mother child 1999; 37: 51-3

5. Haley RW, Schaberg DR et al - 'Extra charges and prolongation of stay attributable to nosocomial infections: a prospective intrahospital comparision' Am. J. Med. 1981; 70: 51-8

6. Heydemann JS, Nelson CL et al - 'Short term preventive antibiotics'- Cli. Orthop.1986; 205:184-7

7. Classen DC, Evans RS et al - 'The timing of prophylactic administration of antibiotics and risk of surgical wound infection' - N Engl Jour Med 1992 Jan 30; 326(5):337-9

8. Creamer E., Cunny RJ et al - 'Sixteen years surveillance of surgical sites in an irish acute care hospital' - Infec control hosp Epedemiol 2002; 23:36 - 40

9. McDonald LC, Yu HT et al - 'Use and abuse of surgical antibiotic prophylaxis in hospitals in Taiwan' - J Formos Med Assos 2001; 100: 5-13

10. Wenzel RP - 'Preoperative antibiotic prophylaxis' - N Engl J Med 1992; 326:337-9

11. Bergamini TM., and MC Polk - 'The importance of tissue antibiotic activity in the prevention of operative wound infection'- J Antimicrob 1989;23:301-313

12. Fritsche TR, Sader HS et al -'Comparitive activity and spectrum of broad-spectrum f3-lactams tested against 12.295 staphylococci and stretrococci: report from the sentry antimicrobial surveillance program' - Diagn Microbiol Infect Dis 2003; 47(2):435-40

13. Woodfield JC, et al - ' A comparison of the prophylactic efficacy of ceftiaxone and cefotaxime in abdominal surgery' - Am J Surg 2002;185:45-9

14. Sabiston Textbook of Surgery, Biologic basis of surgical practice. $17^{\text {th }}$ edition WB Saunders Philadelphia Pg. 
15. Perry TR, Schentag JJ -'Clinical use of ceftriaxone a pharmacokineticpharmacodynamic perspective on the impact of minimum inhibitory concentration and serum protein binding' Clinical Pharmacokinetics - Volume 40, 2001, pp.685694(10)

16. Knight R, Charbonneau P, Ratzer E et al'Prophylatice antibiotics are not indicated in clean general surgery cases' - Am J Surg 2001;182:682-6

17. Classen DC, Evans RS et al-' The timing of prophylactic administration of antibiotics and the risk of surgical-wound infection' $-\mathrm{N}$ Engl J Med 1992; 30:326(5):337-9

18. Wattal C, Oberoi JK - 'Recent Advances in control and prophylaxis of surgical infections, recent advances in surgery-10, Roshan Lal Gupta' - Jaypee brothers, New Delhi 2006. Pg. 76-104

19. Mary T.Hawn, Josna S., et al -'Timing of surgical antibiotic prophylaxis and the risk of surgical site infection' - JAMA Surg 2013;148(7):649-657

20. Brij B. Agrawal -' Prophylactic antibiotic in surgery' - JISMA 2013;26:207

21. J.Wesley Alexander et al -'Updated recommendation for control of surgical site infections' - Annals of Surgery 2011;253(6):1082-1093

22. H. LLenva, J.R.Khambholja -'Recent Advances of antibiotics in clean surgical prophylaxis vs conventional recommended 3 doses of antibiotics' - Gujarat Medical Journal, Aug 2014;69:412:96-98 